Pacific Journal of Mathematics

UNIQUENESS OF EXTENSIONS OF POSITIVE LINEAR 


\title{
UNIQUENESS OF EXTENSIONS OF POSITIVE LINEAR FUNCTIONS
}

\author{
Richard C. Metzler
}

\begin{abstract}
In this paper necessary and sufficient conditions that every approximated function has a unique maximal approximated extension are given. When applied to the Choquet situation this gives a new approach to known uniqueness results for representing measures.
\end{abstract}

Extensions of positive linear functions preserving a certain approximation property were studied in a previous paper [4]. This led to a unified approach to integration theory and the ChoquetBishop-de Leeuw theorem.

Notation will be that of [4]. In particular $V$ and $Y$ will designate ordered vector spaces; $G$ will be a subspace of $V ; W$ will be a wedge in $V$ such that $G \subset W \subset G+V^{+}$and $\alpha$ will be a positive linear function from $G$ to $Y$.

If $f \in V$ and $A \subset V$ we say that $f \alpha$-dominates $A$ if, for every $g$ in $A$ such that $g \leqq f$, the following holds; for every pair $y, z$ in $Y$ such that $y \geqq \alpha\left(f-V^{+}\right)$and $z \leqq \alpha\left(g+V^{+}\right)$we have $y \geqq z$. This is the condition of Theorem 2.1 in [4]. If $\underline{\alpha}(f)=\sup \{\alpha(h): f \geqq h \in G\}$ and $\bar{\alpha}(g)=\inf \{\alpha(h): g \leqq h \in G\}$ both exist in $Y$, this condition is equivalent to the requirement $\underline{\alpha}(f) \geqq \bar{\alpha}(g)$. Now the proof of Theorem 2.1 can be modified easily to yield the following: If $f \in W$ and $f \alpha$-dominates $(-W)$ then $f$ is in $\operatorname{dmn} \underline{\alpha}$ if and only if all maximal $W$-extensions are defined and give the same value on $f$. If $Y$ is assumed Dedekind complete the converse holds; the equivalence implies that $f \alpha$-dominates $(-W)$.

We define a "closure", $G_{1}$, of a subspace $G \subset V$ as $G_{1}=\{f \in V$ : $\exists g \in G^{+}$with $\left(f-\lambda g+V^{+}\right) \cap\left(f+\lambda g-V^{+}\right) \cap G \neq \varnothing$ for all $\left.\lambda>0\right\}$. It is easy to see that $G_{1}$ is a subspace containing $G$. If $G$ contains an order unit $u$, then $G_{1}$ is just the closure of $G$ in the order-unit normed space $\left(\boldsymbol{R} u+V^{+}\right) \cap\left(\boldsymbol{R} u-V^{+}\right)$.

If $f \in V$ and $A, B \subset V$ we say that $A$ separates $f$ and $B$ if, for each $g \in B$, there exists $h \in A$ such that $f \geqq h \geqq g$.

THEOREM 1. Let $Y$ be an Archimedean space. If $f \in W$ is such that $G_{1}$ separates $f$ and $(-W) \cap\left(f-V^{+}\right)$then $f \alpha$-dominates $(-W)$ for every $\alpha$. Consequently if the separation holds for all $f \in W$ then every $\alpha$ has a unique maximal W-extension.

Proof. Suppose $f \geqq g \in(-W)$. By hypothesis there exists $h \in G_{1}$ 
such that $f \geqq h \geqq g$. By definition of $G_{1}$ there is $p \in G^{+}$such that, for any $\delta>0$, there is $q_{\delta} \in G$ with $h-\delta p \leqq q_{\delta} \leqq h+\delta p$. Then $q_{\delta}+\delta p \in\left(g+V^{+}\right) \cap G$ so if $z \leqq \alpha\left(g+V^{+}\right)$we have $z \leqq \alpha\left(q_{\delta}+\delta p\right)$. Similarly if $y \geqq \alpha\left(f-V^{+}\right)$we find $y \geqq \alpha\left(q_{\dot{\delta}}-\delta p\right)$. Then $z-y \leqq$ $2 \delta \alpha(p)$ and the Archimedean property of $Y$ gives $y \geqq z$.

Now we wish to investigate under what conditions the unique extension property implies the $G_{1}$ separation of Theorem 1 .

Lemma. Assume $G$ has an order unit $u$ and suppose $Y^{+} \neq\{0\}$. If every $\alpha$ has a unique maximal $W$-extension then, for every $f \in W \cap$ $\left(G-V^{+}\right)$and $\varepsilon>0, G$ separates $f+\varepsilon u$ and $(-W) \cap\left(f-V^{+}\right) \cap$ $\left(G+V^{+}\right)$.

Proof. We will suppose that there is $f \in W \cap\left(G-V^{+}\right)$and $\varepsilon>0$ such that $G$ does not separate $f+\varepsilon u$ and $(-W) \cap\left(f-V^{+}\right) \cap\left(G+V^{+}\right)$. Then there exists $g \in(-W) \cap\left(f-V^{+}\right) \cap\left(G+V^{+}\right)$such that if $A=$ $\{h \in G: h \geqq g+(\varepsilon / 2) u\}, B=\{h \in G: h \leqq f+\varepsilon u\}$ and $U=\{h \in G:-(\varepsilon / 2) u \leqq$ $h \leqq(\varepsilon / 2) u\}$ then $(A+U) \cap B=\varnothing$. Since $U$ is radial at the origin as a subset of $G$ a standard separation result [3; p. 23] shows that there exists a linear functional $\phi$ on $G$ which strongly separates $A$ and $B$. By taking $-\phi$ if necessary we can assume that $r_{0}=\sup \{\varphi(p)$ : $p \in B\}<\inf \{\varphi(q): q \in A\}=s_{0}$. Now let $p \in G^{+}$. Then if $f_{1} \in B$ and $f_{2} \in A$ we have $\varphi\left(f_{2}+r p\right)=\varphi\left(f_{2}\right)+r \varphi(p) \geqq \varphi\left(f_{1}\right)$ for all $r \geqq 0$. This shows that $\varphi(p) \geqq 0$ and we see that $\varphi$ is a positive linear functional on $G$. Then we have $\varphi(f+\varepsilon u)=r_{0}<s_{0}=\bar{\varphi}(g+(\varepsilon / 2) u)$. Since $g+(\varepsilon / 2) u \epsilon$ $(-W)+G \subset(-W)$ we see that $f+\varepsilon u$ does not $\varphi$-dominate $(-W)$. Since $\boldsymbol{R}$ is Dedekind complete Theorem 2.1 of [4] shows that $\varphi$ does not have a unique maximal $W$-extension. Now choose $y>0$ in $Y$ and define $\alpha: G \rightarrow Y$ by $\alpha(f)=\varphi(f) y$. Then it is easy to see that $\alpha$ does not have a unique maximal $W$-extension.

THEOREM 2. In addition to the assumptions of the lemma we assume that $V$ is Dedekind $\sigma$-complete and $W$ is closed under finite infs. Then, if every $\alpha$ has a unique maximal $W$-extension, $G_{1}$ separates $f$ and $\left(f-V^{+}\right) \cap(-W)$ for all $f \in W$.

Proof. Given any $f \in W$ and $g \leqq f$ such that $g \in-W$ we wish to show that $G_{1}$ separates $f$ and $g$. Now we can assume, without loss of generality, that $f \in G-V^{+}$and $g \in G+V^{+}$. If this were not so we could choose $g^{\prime} \in G \cap\left(f-V^{+}\right)$and (using the assumptions that $W$ is closed under finite infs and $\left.W \subset G+V^{+}\right) f^{\prime} \in G \cap\left(g \vee g^{\prime}+V^{+}\right)$. Then we could replace $f$ by $f \wedge f^{\prime}$ and $g$ by $g \vee g^{\prime}$. Clearly any element which separates $f \wedge f^{\prime}$ and $g \vee g^{\prime}$ will separate $f$ and $g$.

We adapt a technique of Edwards [2] to find $h \in G_{1}$ such that 
$f \geqq h \geqq g$. Let $g_{0}=g-u$ and $f_{0}=f+u$ and use the lemma to choose $h_{0} \in G$ such that $g_{0} \leqq h_{0} \leqq f_{0}$. Now assume that for $m=$ $1,2, \cdots, n$ we have $f_{m} \in W, g_{m} \in-W$ and $h_{m} \in G$ such that $g-2^{-m} u \leqq$ $g_{m} \leqq h_{m} \leqq f_{m} \leqq f+2^{-m} u$ and $-3 \cdot 2^{-m-1} u \leqq h_{m}-h_{m-1} \leqq 3 \cdot 2^{-m-1} u$.

Let

$$
g_{n+1}=\left(g-2^{-n-1} u\right) \vee\left(h_{n}-3 \cdot 2^{-n-2} u\right) \in-W
$$

while

$$
\begin{aligned}
f_{n+1} & =\left(f+2^{-n-1} u\right) \wedge\left(h_{n}+3 \cdot 2^{-n-2} u\right) \\
& =\left(f+2^{-n-2} u\right) \wedge\left(h_{n}+2^{-n-1} u\right)+2^{-n-2} u \in W .
\end{aligned}
$$

Now $g_{n+1}+2^{-n-2} u \leqq f_{n+1}$ results from the following inequalities:

$$
\begin{gathered}
g-2^{-n-1} u \leqq f+2^{-n-2} u ; g-2^{-n-1} u \leqq h_{n}+2^{-n-1} u ; \\
h_{n}-3 \cdot 2^{-n-2} u \leqq f+2^{-n-2} u ; \text { and } h_{n}-3 \cdot 2^{-n-2} u \leqq h_{n}+2^{-n-1} u .
\end{gathered}
$$

Hence we can use the lemma to choose $h_{n+1} \in G$ such that $g$ $2^{-n-1} u \leqq g_{n+1} \leqq h_{n+1} \leqq f_{n+1} \leqq f+2^{-n-1} u$ and $-3 \cdot 2^{-n-2} u \leqq g_{n+1}-h_{n} \leqq$ $h_{n+1}-h_{n} \leqq f_{n+1}-h_{n} \leqq 3 \cdot 2^{-n-2} u$. This completes the inductive definition.

Now $-3 \cdot 2^{-n-2} u \leqq h_{n+1}-h_{n} \leqq 3 \cdot 2^{-n-2} u$ implies $-3 \cdot 2^{-m-1} u \leqq h_{p}-$ $h_{m} \leqq 3 \cdot 2^{-m-1} u$ for all $p \geqq m$.

Now let $h=\inf _{n}\left(\sup _{k \geqq n} h_{k}\right)$ which exists by the inequality for $h_{p}-h_{m}$ and the fact that $V$ is Dedekind $\sigma$-complete. From the inequalities $g-2^{-n-1} u \leqq h_{n+1} \leqq f+2^{-n-1} u$ we conclude, since a Dedekind $\sigma$-complete space is Archimedean, that $g \leqq h \leqq f$. Since we can replace $h_{p}$ by $h$ in the inequality for $h_{p}-h_{m}$ we see that $h \in G_{1}$ as desired.

Now in the approach to Choquet boundary theory given in [4] we assume that $V$ is the space of continuous functions on a compact Hausdorff space $X, G$ is a closed subspace and $W$ is a wedge of bounded continuous functions on $X$ closed under finite infs. Then $G=G_{1}$ and, since $W$-approximated linear functionals are maximal measures, we see that uniqueness of representing "Choquet" measures implies the separation of Theorem 2. This gives the "geometric simplex" result of Boboc and Cornea [1, Th. 4]. If we let $X$ be a convex compact subset of a locally convex space, $G$ the continuous affine functions and $W$ the wedge of finite infs from $G$ then we find that the separation property reduces in this case to the interpolation version of the Riesz decomposition property. This gives the "Choquet simplex" result of Edwards [2].

We now investigate an alternate characterization of the space $G_{1}$. We define $G_{Y}$ to be the largest subspace of $V$ such that every positive linear $\alpha: G \rightarrow Y$ has a unique positive linear extension to $G_{Y}$. In the notation of [4] we can write $G_{Y}$ as $\cap\left\{\mathrm{dmn} \alpha_{G}: \alpha\right.$ positive 
and linear from $G$ to $Y$ \}.

THEOREM 3. If $Y$ is Dedekind $\sigma$-complete $G_{1} \subset G_{Y}$.

Proof. If $f \in G_{1}$ then there exists $g \in G^{+}$and a sequence $\left\{h_{n}\right\} \subset G$ such that $h_{n}-(1 / n) g \leqq f \leqq h_{n}+(1 / n) g$ for all $n$. This gives us

$$
-\left(\frac{1}{n}+\frac{1}{m}\right) g \leqq h_{n}-h_{m} \leqq\left(\frac{1}{n}+\frac{1}{m}\right) g
$$

for all $n$ and $m$. Now let $\alpha$ be any positive linear function from $G$ to $Y$. Then

$$
-\left(\frac{1}{n}+\frac{1}{m}\right) \alpha(g) \leqq \alpha\left(h_{n}\right)-\alpha\left(h_{m}\right) \leqq\left(\frac{1}{n}+\frac{1}{m}\right) \alpha(g)
$$

gives $-(1 / m) \alpha(g) \leqq y-\alpha\left(h_{m}\right) \leqq(1 / m) \alpha(g)$ for $y=\inf _{n}\left(\sup _{k \geqq n} \alpha\left(h_{k}\right)\right)$. Then $h_{n}-(1 / n) g \leqq f \leqq h_{n}+(1 / n) g$ for all $n$ implies $\bar{\alpha}(f) \leqq y \leqq \underline{\alpha}(f)$. From this it is not hard to see that every maximal positive extension of $\alpha$ assumes the value $y$. Since $\alpha$ was arbitrary we conclude that $f \in G_{Y}$.

THEOREM 4. If $G$ has an order-unit and $Y^{+} \neq\{0\}$ then $G_{Y} \subset G_{1}$.

Proof. Note first that if $f \in G_{Y}$ we must have $f \in\left(G-V^{+}\right) \cap$ $\left(G+V^{+}\right)$. For if $f$ is not in $G-V^{+}$let $\hat{\alpha}$ be a maximal positive extension of a positive linear $\alpha$ from $G$ to $Y$. Choose $y>0$ in $Y$ and define $\alpha_{1}$ by $\alpha_{1}(g+r f)=\alpha(g)+r(\hat{\alpha}(f)+y)$. Then since $\hat{\alpha}$ is positive and $f \notin G-V^{+}$it is easy to see that $\alpha_{1}$ is positive. Then any maximal extension of $\alpha_{1}$ contradicts $f \in G_{Y}$. A symmetric argument gives $f \in G+V^{+}$.

Now if $f \in G_{Y}$ let $W=G+R f$. Then the lemma applies and we can assume that $G$ separates $f+\varepsilon u$ and $(-W) \cap\left(f-V^{+}\right) \cap\left(G+V^{+}\right)$ for all $\varepsilon>0$. Since $f \in(W) \cap(-W) \cap\left(f-V^{+}\right) \cap\left(G+V^{+}\right)$we see there exists $h_{\varepsilon} \in G$ such that $f \leqq h_{\varepsilon} \leqq f+\varepsilon u$ for all $\varepsilon>0$. Hence $f \in G_{1}$ as desired.

\section{REFERENCES}

1. N. Boboc and A. Cornea, Cônes des fonctions continues sur un espace compact, C. R. Acad. Sc. Paris, t., 261 (October 4, 1965), 2564-2567.

2. D. A. Edwards, Séparation des fonctions réeles definies sur un simplexe de Choquet, C. R. Acad. Sc. Paris, t., 261 (October 1965), 2798-2800.

3. J. Kelley and I. Namioka, Linear Topological Spaces, D. Van Nostrand Co., Inc., Princeton, New Jersey, (1963).

4. R. C. Metzler, Positive linear functions, integration, and Choquet's theorem, Pacific J. Math., 60 (1975), 277-296.

Received June 24, 1977.

The University of New Mexico

ALBUQUERQUE, NM 87131 


\section{PACIFIC JOURNAL OF MATHEMATICS}

\section{EDITORS}

RICHARD ARENS (Managing Editor)

University of California

Los Angeles, California 90024

C. W. Curtis

University of Oregon

Eugene, OR 97403

C. C. MOORE

University of California

Berkeley, CA 94720

\section{J. DUGUNDJI}

Department of Mathematics University of Southern California Los Angeles, California 90007

R. Finn AND J. Milgram Stanford University Stanford, California 94305

ASSOCIATE EDITORS
E. F. BECKENBACH

B. H. NeUMANN

F. WOLF

K. YoshidA

\section{SUPPORTING INSTITUTIONS}

UNIVERSITY OF BRITISH COLUMBIA UNIVERSITY OF SOUTHERN CALIFORNIA CALIFORNIA INSTITUTE OF TECHNOLOGY STANFORD UNIVERSITY UNIVERSITY OF CALIFORNIA MONTANA STATE UNIVERSITY UNIVERSITY OF TOKYO UNIVERSITY OF NEVADA, RENO UNIVERSITY OF UTAH NEW MEXICO STATE UNIVERSITY OREGON STATE UNIVERSITY UNIVERSITY OF OREGON

WASHINGTON STATE UNIVERSITY UNIVERSITY OF WASHINGTON OSAKA UNIVERSITY 


\section{Pacific Journal of Mathematics}

\section{Vol. 74, No. $1 \quad$ May, 1978}

Gerald Arthur Anderson, Computation of the surgery obstruction groups

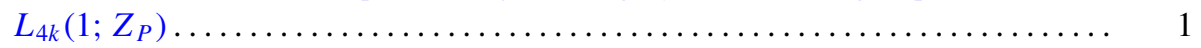

R. K. Beatson, The degree of monotone approximation ................ 5

Sterling K. Berberian, The character space of the algebra of regulated functions . . . 15

Douglas Michael Campbell and Jack Wayne Lamoreaux, Continua in the plane with

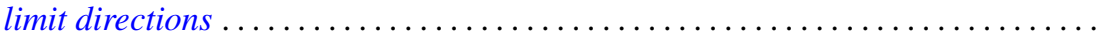

R. J. Duffin, Algorithms for localizing roots of a polynomial and the Pisot

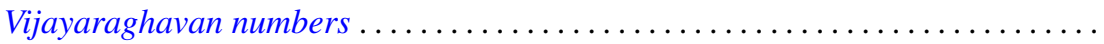

Alessandro Figà-Talamanca and Massimo A. Picardello, Functions that operate on

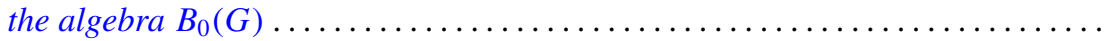

John Erik Fornaess, Biholomorphic mappings between weakly pseudoconvex

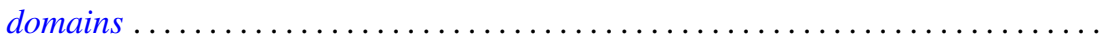

Andrzej Granas, Ronald Bernard Guenther and John Walter Lee, On a theorem of S.

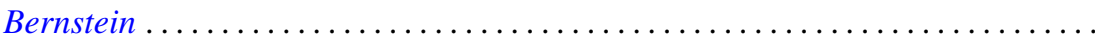

Jerry Grossman, On groups with specified lower central series quotients . .........

William H. Julian, Ray Mines, III and Fred Richman, Algebraic numbers, a constructive development . . . . . . . . . . . . . . . . . . . . . . .

Surjit Singh Khurana, A note on Radon-Nikodým theorem for finitely additive

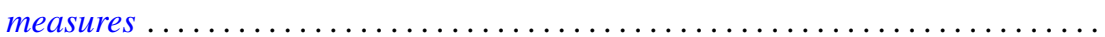

Garo K. Kiremidjian, A Nash-Moser-type implicit function theorem and nonlinear

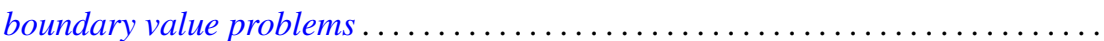

Ronald Jacob Leach, Coefficient estimates for certain multivalent functions ....

John Alan MacBain, Local and global bifurcation from normal eigenvalues. II . . 133

James A. MacDougall and Lowell G. Sweet, Three dimensional homogeneous algebras...

John Rowlay Martin, Fixed point sets of Peano continua ......

R. Daniel Mauldin, The boundedness of the Cantor-Bendixson order of some analytic sets...

Richard C. Metzler, Uniqueness of extensions of positive linear functions ..

Rodney V. Nillsen, Moment sequences obtained from restricted powers . .

Keiji Nishioka, Transcendental constants over the coefficient fields in differential elliptic function fields...

Gabriel Michael Miller Obi, An algebraic closed graph theorem

Richard Cranston Randell, Quotients of complete intersections by $\mathbf{C}^{*}$ actions . . 221

Bruce Reznick, Banach spaces which satisfy linear identities . .

Bennett Setzer, Elliptic curves over complex quadratic fields...

Arne Stray, A scheme for approximating bounded analytic functions on certain subsets of the unit disc.

Nicholas Th. Varopoulos, A remark on functions of bounded mean oscillation and bounded harmonic functions. Addendum to: "BMO functions and the

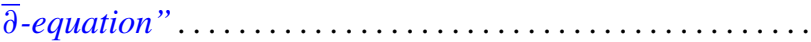

Charles Irvin Vinsonhaler, Torsion free abelian groups quasi-projective over their

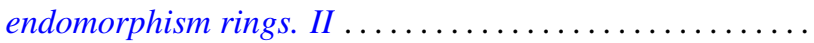

Thomas R. Wolf, Characters of $p^{\prime}$-degree in solvable groups ... 